\title{
Combined and intravenous administration of TXA reduces blood loss more than topical administration in primary total knee arthroplasty: A randomized clinical trial
}

\author{
Jiri Lostak ${ }^{\mathrm{a}}$, Jiri Gallo ${ }^{\mathrm{a}}$, Lubos Balaz ${ }^{\mathrm{a}}$, Jana Zapletalova ${ }^{\mathrm{b}}$
}

\begin{abstract}
Aim. To determine the most effective administration of tranexamic acid (TXA) in patients with primary total knee arthroplasty (TKA).

Material and Method. We enrolled a total of 400 patients (154 men and 346 women) in this randomized trial (4 groups, each of 100 patients). The first group (IV1) had a single intravenous dose (15 mg TXA/kg) prior to skin incision. Group 2 (IV2) had TXA in 2 intravenous doses (15 mg TXA/kg): prior to skin incision and 6 hours after the first dose. Group 3 (TOP) had $2 \mathrm{~g}$ TXA in $50 \mathrm{~mL}$ of saline irrigated topically at the end of the surgery. The fourth group (COMB) combined IV1 and TOP regimens. We monitored the amount of total blood loss (TBL), haemoglobin drop, use of blood transfusions (BTs), and complications in each patient.

Results. The amount of TBL was significantly lower in IV1, IV2 and COMB regimens compared to the TOP $(P<0.0001)$. The lowest decrease in haemoglobin within 12 hours after surgery was observed in intravenous regimens $(P=0.045)$. A significant difference in haemoglobin decrease on day 1 after the surgery was demonstrated in the COMB and intravenous regimens $(P=0.011)$.

Conclusion. In primary TKA, it is preferable to administer TXA intravenously in two doses or in a combined regimen. Simple topical administration of TXA was not as effective and is indicated only in cases where systemic administration of TXA is contraindicated. No substantial complications occurred in either group of patients.
\end{abstract}

Key words: total knee arthroplasty, tranexamic acid, topical administration, intravenous administration, combined administration, randomized clinical trial

Received: April 3, 2020; Revised: September 27, 2020; Accepted: September 29, 2020; Available online: October 15, 2020 https://doi.org/10.5507/bp.2020.045

(c) 2022 The Authors; https://creativecommons.org/licenses/by/4.0/

${ }^{a}$ Department of Orthopaedics, University Hospital Olomouc and Faculty of Medicine and Dentistry, Palacky University Olomouc, Czech Republic

${ }^{b}$ Department of Medical Biophysics, Faculty of Medicine and Dentistry, Palacky University Olomouc, Czech Republic Corresponding author: Jiri Gallo, e-mail:jiri.gallo@volny.cz

\section{INTRODUCTION}

Tranexamic acid (TXA) can be administered intravenously, orally or directly into the joint via irrigation in the perioperative period for primary total knee arthroplasty (TKA) (ref. ${ }^{1}$ ). Most studies have been performed with systemic and topical administration of TXA (ref. ${ }^{1,2}$ ). However, there are still concerns about the potential risk of thromboembolic disease in the case of intravenous administration in higher doses ${ }^{3,4}$. For this reason, some studies favour topical administration with the aim of transferring the maximum effect to the target area and thus avoid an overall effect on the fibrinolytic system ${ }^{5,6}$. It is potentially most effective to combine both routes, to reduce the intravenous dose, thereby minimizing the risk of thromboembolism and increase the effect by additional topical administration ${ }^{4,5,7}$.

Some studies suggest that combined TXA administration have a greater effect than single topical or intravenous administration $^{8,9}$. TKA appears to be an ideal candidate for combined procedures due to the large bleeding area. However, there are concerns about the synergy of the systemic and topical TXA effect. In our previous study, we determined the safety profile for systemic, combined and topical administration of TXA in patients undergoing primary TKA $\left(\right.$ ref. $\left.{ }^{10}\right)$. We found no significant effect on systemic markers of fibrinolysis in either regimen. Although many excellent studies have focused on search for the optimal protocol for use of TXA in TKA, differences in views on the optimal regimen of TXA administration have only increased ${ }^{1,2,8}$. Even large-scale randomized studies and meta-analyses do not provide consistent conclusions on the proper administration of TXA in terms of dose or number of doses ${ }^{11}$.

The primary objective of our study was to determine which route of TXA administration has the greatest effect on the reduction of blood loss (into the drainage system, hidden loss) and use of blood transfusions. We compared postoperative haemoglobin and its decrease in the early postoperative period in individual regimens. 


\section{MATERIAL AND METHOD}

\section{Patient cohort}

We enrolled a total of 400 consecutive patients into this prospective randomized trial. These were indicated for primary TKA in our department between June 2018 and October 2019. The patients received TXA during the perioperative period according to a predetermined protocol. Each group consisted of 100 patients (Fig. 1). The inclusion criteria for the enrolment in the trial were normal preoperative blood count (haemoglobin, platelets) and blood coagulation (INR, Quick, aPTT). The trial did not include patients with a history of any disease associated with blood coagulation defects, with a history of venous thromboembolism (VTE), severe kidney disease or convulsions. The basic characteristics of the 4 groups were similar (Table 1). A more detailed analysis of BMI (comparing cohorts in pairs) showed no significant difference (results of Dunn's post hoc test).

The clinical register of joint replacements has been approved by the hospital management, and its administration is regulated by an amended ethical and legal protocol. The local Ethics Committee approved the study in accordance with the latest Helsinki Declaration; all the enrolled patients agreed with the use of anonymized data for research purposes (registration number 87-67). Prior to enrolment into the study, patients signed an informed consent.

\section{Collection of preoperative data}

Data were collected prospectively according to a predetermined procedure. The medical data were registered by the physician during the admission interview. The physician also recorded the basic medical history, including the primary diagnosis and results of clinical examination.

\section{Preoperative preparations}

Patient preparation procedures were initiated on the day of admission, i.e. one day before the surgery. In the context of VTE prevention, we applied either lowmolecular-weight heparin (Fraxiparine; Aspen Pharma Trading Limited, Dublin, Ireland), first administered subcutaneously $12 \mathrm{~h}$ before the surgery (at the dose recommended by the manufacturer). Alternatively, Rivaroxaban (Xarelto; Bayer AG, Leverkusen, Germany) was administered orally 6 to $8 \mathrm{~h}$ after the surgery. Postoperative infection prevention largely consisted in i.v. administration of $1 \mathrm{~g}$ of the third-generation cephalosporin antibiotic (Azepo; Sandoz, Holzkirchen, Germany). Clindamycin $600 \mathrm{mg}$ i.v. was administered in case of allergy (Fresenius Kabi, Bad Homburg vor der Höhe, Germany). The antibiotic was administered intravenously 30 to $60 \mathrm{~min}$ before the skin incision. The additional doses of antibiotics were administered 8 and $16 \mathrm{~h}$ after the first administration.

\section{Surgical procedure and the implant}

The surgical procedure was performed under general or block anaesthesia. The procedure invariably used skin

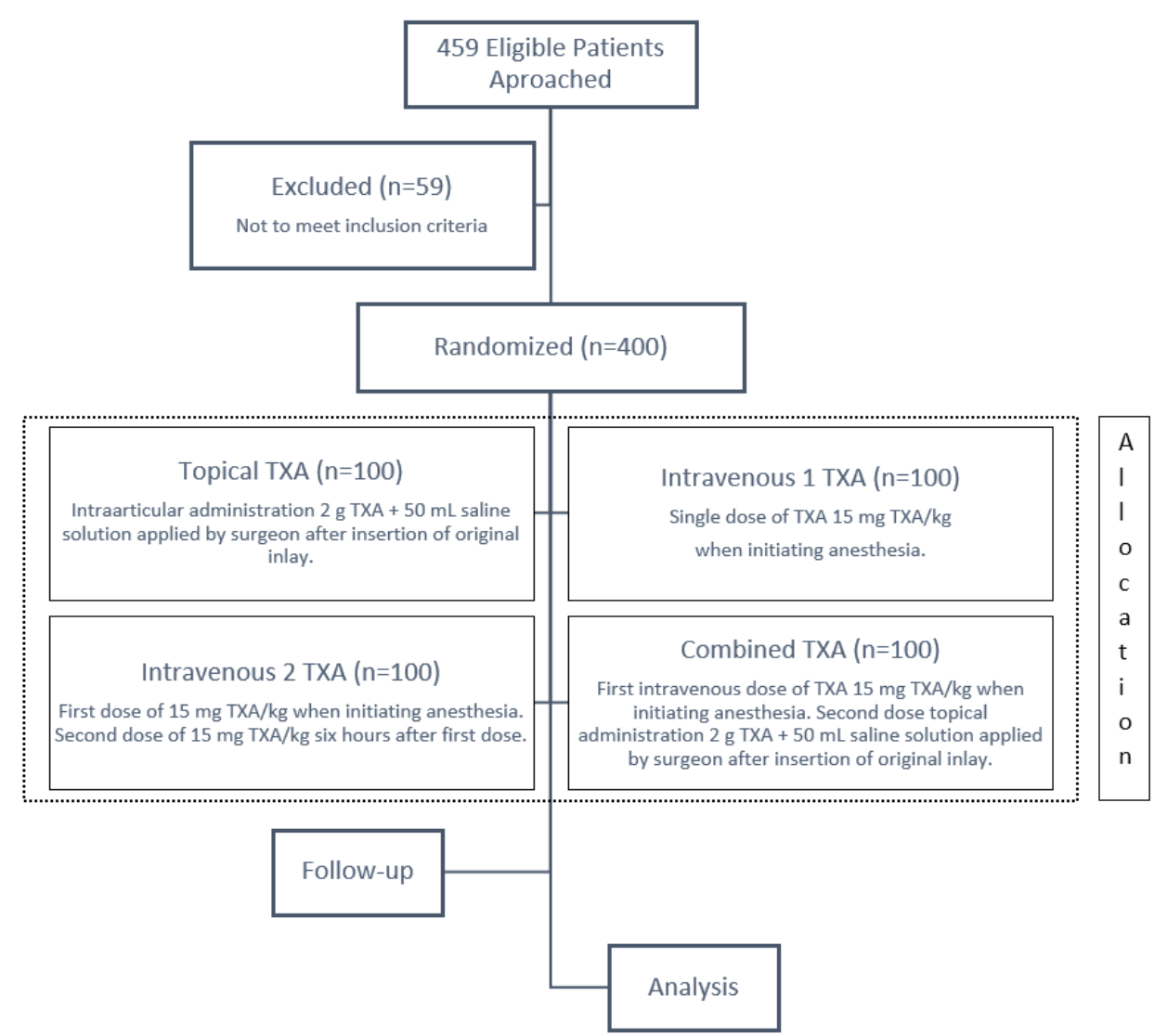

Fig. 1. Flow chart showing study subject identification, inclusion and exclusion, allocation and protocols of TXA. 
Table 1. Comparison of the groups in their basic parameters.

\begin{tabular}{lccccc}
\hline & COMB & IV1 & IV2 & TOP & $P$ \\
\hline \# of patients & 100 & 100 & 100 & 100 & - \\
Primary osteoarthritis & $89 / 11$ & $89 / 11$ & $88 / 12$ & $87 / 13$ & 0.966 \\
Gender (m/f) & $38 / 62$ & $33 / 67$ & $36 / 64$ & $47 / 53$ & 0.203 \\
Mean age \pm SD & $68.9 \pm 6.9$ & $69.1 \pm 7.6$ & $69.2 \pm 7.7$ & $71.4 \pm 6.4$ & 0.064 \\
BMI $\left(\mathrm{kg} / \mathrm{m}^{2}\right.$ ) Median (min-max) & $32.3(20.9-42.3)$ & $31.2(23.3-53.4)$ & $40.4(21.3-48.8)$ & $30.4(23.2-44.7)$ & 0.028 \\
Patient type A/B/C* & $57 / 40 / 3$ & $64 / 35 / 1$ & $67 / 33 / 0$ & $58 / 39 / 3$ & 0.432 \\
X-rays K-L II/III/IV & $1 / 94 / 5$ & $1 / 94 / 5 /$ & $3 / 94 / 3$ & $1 / 96 / 3$ & 0.870 \\
X-rays IKDC C/D/O & $47 / 46 / 7$ & $52 / 41 / 7$ & $46 / 48 / 6$ & $50 / 47 / 3$ & 0.817 \\
CCI median (min-max) & $2(0-14)$ & $1(0-14)$ & $0(0-14)$ & $2(0-14)$ & $<0.0001$ \\
\hline
\end{tabular}

CCI, Charlson Comorbidity Index; TOP, topical administration of TXA; COMB, combination of topical and intravenous administration of TXA; IV1, single intravenous administration; IV2, two intravenous doses of TXA; SD, standard deviation; m, male; f, female; K-L, Kellgren-Lawrence classification; IKDC, International Knee Documentation Committee; $P$, significance; *According to the degree of risk of infection $(\mathrm{A}=$ minimal risk; $\mathrm{B}=$ moderate; $\mathrm{C}=$ highest; modified from McPherson et al. ${ }^{12}$ )

Table 2. Overview of perioperative blood loss.

\begin{tabular}{|c|c|c|c|c|c|}
\hline & COMB & IV1 & IV2 & TOP & $P$ \\
\hline Total blood loss into drains $(\mathrm{mL})$ median & 450 & 375 & 350 & 635 & $<0.0001$ \\
\hline Hidden blood loss (mL) & 192 & 196 & 178 & 275 & 0.015 \\
\hline Blood loss during surgery & 300 & 300 & 300 & 300 & 0.268 \\
\hline Total blood loss $(\mathrm{mL}),($ during surgery + into drainage system $)$ & 750 & 650 & 605 & 900 & $<0.0001$ \\
\hline Maximum blood loss to drainage system & 1500 & 1400 & 1500 & 1790 & - \\
\hline Minimum blood loss to drainage system & 0 & 0 & 0 & 0 & - \\
\hline $\begin{array}{l}\text { Total post-operative blood loss into drains including hidden } \\
\text { blood loss }\end{array}$ & 620 & 608 & 534 & 861 & $<0.0001$ \\
\hline Total blood loss including hidden loss (mL) & 979 & 919 & 819 & 1185 & $<0.0001$ \\
\hline
\end{tabular}

Median values for the data are provided in the table because blood losses did not have a normal distribution (verified by the Shapiro-Wilk test). TOP, topical administration of TXA; COMB, combination of topical and intravenous administration of TXA; IV1, single intravenous administration; IV2, two intravenous doses of TXA; $P$, significance.

Table 3. Comparison of blood loss in individual TXA regimens (results of Dunn's post hoc test).

\begin{tabular}{lccccc}
\hline & $\begin{array}{c}\text { Total blood } \\
\text { loss into drains }\end{array}$ & $\begin{array}{c}\text { Hidden blood } \\
\text { loss }\end{array}$ & $\begin{array}{c}\text { Total loss during surgery } \\
\text { and postoperative loss to } \\
\text { drainage systems }\end{array}$ & $\begin{array}{c}\text { Total postoperative loss } \\
\text { to drainage systems } \\
\text { including hidden loss }\end{array}$ & $\begin{array}{c}\text { Total loss including } \\
\text { hidden loss }\end{array}$ \\
\hline TOP vs. COMB & 0.004 & - & 0.021 & 0.004 & 0.015 \\
TOP vs. IV1 & $<0.0001$ & - & 0.004 & $<0.0001$ & 0.005 \\
TOP vs. IV2 & $<0.0001$ & 0.010 & $<0.0001$ & $<0.0001$ & $<0.0001$ \\
\hline
\end{tabular}

TOP, topical administration of TXA; COMB, combination of topical and intravenous administration of TXA; IV1, single intravenous administration; IV2, two intravenous doses of TXA.

incision and the medial parapatellar approach. The bleeding was stopped during the surgery using electrocoagulation. The tourniquet was only activated for cementing (typically up to $15 \mathrm{~min}$ ). All operations were performed by experienced surgeons. We used only cemented implants preserving the posterior cruciate ligament or replacing its function.

\section{TXA protocols}

Each group had a well-defined protocol for TXA administration and dosing (Fig. 1). Group 1 (IV1) included patients with TXA administered by the anaesthesiologist prior to skin incision as a single intravenous dose (15 mg TXA/kg). Group 2 (IV2) included patients with TXA administered in two intravenous doses (15 mg TXA/kg), the first dose as described above (IV1) and the subsequent dose 6 hours after the first administration. Group 3 (TOP) included patients with TXA administered topically by lavage with a diluted solution of $2 \mathrm{~g}$ TXA in $50 \mathrm{~mL}$ of saline. The fourth group (COMB) had TXA administered in combination, the first dose of $15 \mathrm{mg}$ TXA $/ \mathrm{kg}$ intravenously prior to skin incision and the second dose topically via irrigation using the mixture of $2 \mathrm{~g}$ TXA in $50 \mathrm{~mL}$ of saline at the end of the surgery. In all patients, the drains were left closed for $1 \mathrm{~h}$ from the surgery.

\section{Endpoints \\ Blood loss including hidden blood loss}

We evaluated the amount of blood loss during the surgery and postoperative period into the drainage system 
in each patient. Hidden blood loss was calculated using a formula that includes perioperative and postoperative blood loss related to the sex and weight of the patient ${ }^{13}$. Briefly, hidden blood loss $=$ true calculated red blood count (RBC) - total measured RBC volume loss - total measured $\mathrm{RBC}$ volume gain [True calculated $\mathrm{RBC}=\mathrm{PBV}$ (patient blood volume) * (hematocrit before surgery-postoperative); total measured RBC volume loss = intraoperative blood loss + postoperative blood loss; total measured $\mathrm{RBC}$ volume gain $=$ blood reinfused (recuperation $)+\mathrm{RBC}$ volume transfused; $\mathrm{PBV}=\mathrm{k} 1 \times \mathrm{h} 3+\mathrm{k} 2 \times \mathrm{w}+\mathrm{k} 3$; h, height (m); w, weight $(\mathrm{kg})$; for male, $\mathrm{k} 1=0.3669, \mathrm{k} 2=0.03219$, and $\mathrm{k} 3=0.6041$; for female $\mathrm{k} 1=0.3561, \mathrm{k} 2=0.03308$, and $\mathrm{k} 3=0.1833]$.

We also recorded the number of blood transfusions administered, together with the type of transfusion (autotransfusion, allogeneic blood transfusion).

\section{Haemoglobin, haematocrit}

The first blood sample was collected from each patient on the day of admittance to the ward in order to determine the preoperative haemoglobin level. The second sample was collected 4 hours after the surgery. The blood picture was also checked on post-operative day 1 and, in certain patients, also on post-operative day 2 . Since we had available the initial preoperative levels, we were able to determine haemoglobin decrease (difference between the preoperative level and postoperative level) induced by the surgical procedure. The blood picture was routinely examined on a Sysmex XN 3000 analyser (Sysmex, Kobe, Japan).

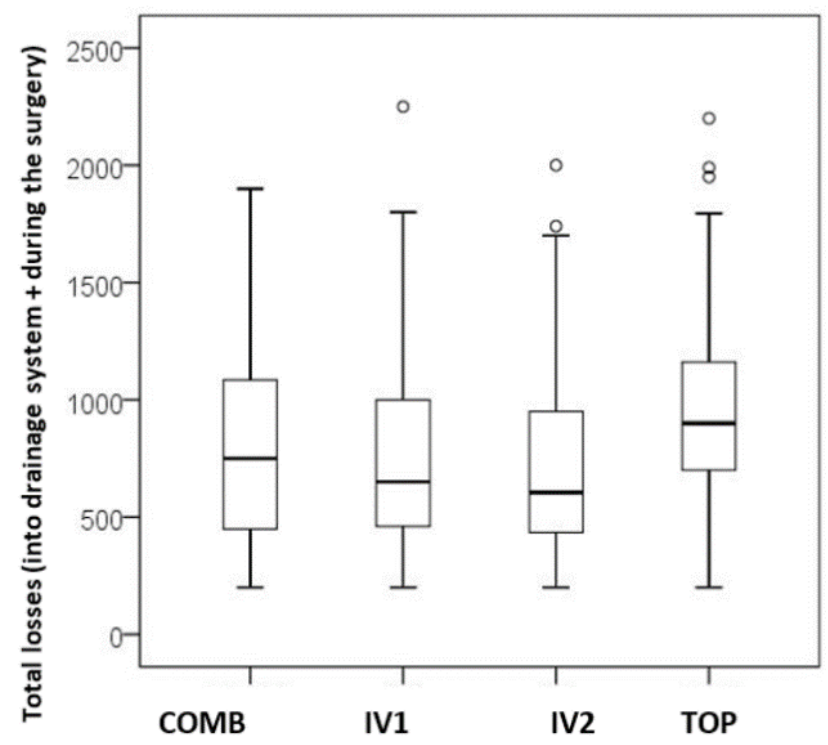

Fig. 2. Graphical representation of the range of blood loss during surgery and postoperative period into drainage systems in individual groups.

TOP, topical administration of TXA; COMB, combination of topical and intravenous administration of TXA; IV1, single intravenous administration; IV2, two intravenous doses of TXA.

\section{Incidence of haematomas, secretion from the wound after $4^{\text {th }}$ post-operative day}

We evaluated the frequency of early postoperative complications (occurrence of haematomas, wound secretion 4 days after the surgery). Upon discharge from the ward, we recorded the incidence, size and location of the haematoma. Secretion from the wound was recorded if persisting after post-operative day 4 .

\section{Statistics}

The distribution of the quantitative data was subjected to the Shapiro-Wilk normality test. Data with a normal distribution are presented as means and standard deviations. ANOVA was used to test differences between the groups for normally distributed data. Data that were not distributed normally are shown as medians and minimum and maximum values and analysed using the non-parametric Kruskal-Wallis test with Dunn's post-hoc correction. Qualitative data are described as absolute and relative frequencies and were analysed by the exact Fisher test. The IBM SPSS Statistics, version 23 (Armonk, NY; IBM Corp.) was used for the analysis. The statistical level of significance was 0.05 in all cases.

\section{RESULTS}

\section{Primary objectives of the trial \\ Blood loss after the surgery}

There was a significant difference in total postoperative blood loss in the following order: IV2, IV1 <COMB $<$ TOP $(P<0.0001)$. On average, the lowest drainage discharge was achieved in intravenous regimens (IV2 $=350$ $\mathrm{mL}$ and $\mathrm{IV} 1=375 \mathrm{~mL}$ ) and combined administration

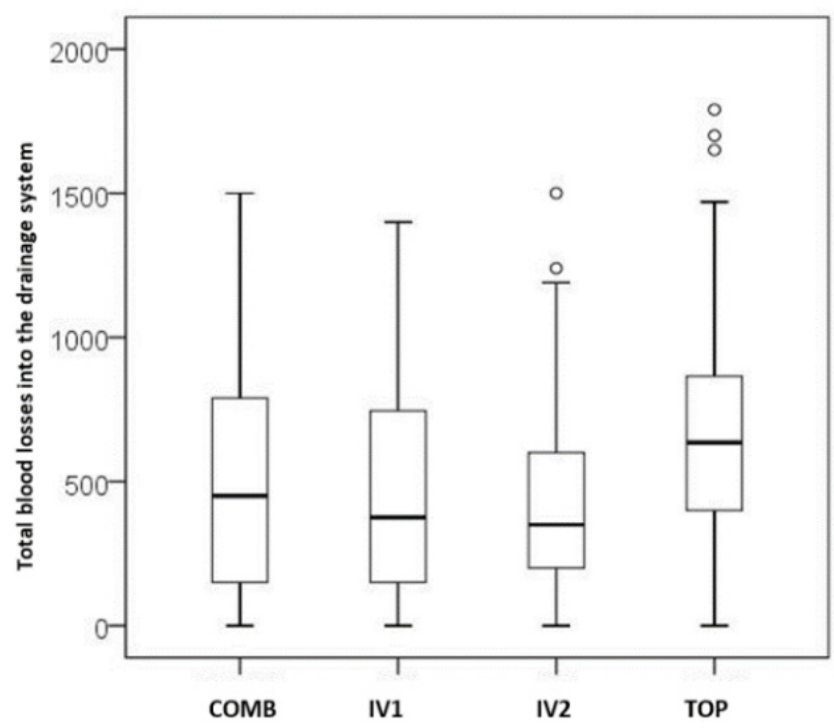

Fig. 3. An illustration of the range of blood loss to drainage systems in each group.

TOP, topical administration of TXA; COMB, combination of topical and intravenous administration of TXA; IV1, single intravenous administration; IV2, two intravenous doses of TXA. 
Table 4. Comparison of haemoglobin level, its decrease.

\begin{tabular}{lccccc}
\hline HAEMOGLOBIN & COMB & IV1 & IV2 & TOP & $P$ \\
\hline Preoperative (average, g/L) & 130 & 131 & 131 & 134 & 0.283 \\
Post-operative 3 h (average, g/L) & 123 & 121 & 122 & 124 & 0.266 \\
Post-operative 6 h (average, g/L) & 123 & 120 & 119 & 125 & 0.256 \\
Post-operative 12 h (average, g/L) & 118 & 115 & 115 & 118 & 0.657 \\
Post-operative day 1. (average, g/L) & 113 & 113 & 113 & 112 & 0.996 \\
Post-operative day 2. & 104 & 104 & 107 & 104 & 0.387 \\
Difference pre-operative - day 1. (average, g/L) & 17 & 18 & 18 & 21 & 0.011 \\
Difference pre-operative - 6 h postop. (average, g/L) & 11 & 10 & 10 & 11 & 0.932 \\
Difference post-operative 3 h - 6 h postop. (average, g/L) & 5 & 1 & 4 & 3 & 0.054 \\
Difference post-operative 6 h - 12 h postop. (average, g/L) & 5 & 5 & 5 & 7 & 0.629 \\
Difference post-operative 3 h - 12 h postop. (average, g/L) & 10 & 5 & 9 & 11 & 0.045 \\
\hline
\end{tabular}

TOP, topical administration of TXA; COMB, combination of topical and intravenous administration of TXA; IV1, single intravenous administration; IV2, two intravenous doses of TXA; $P$, significance.

Table 5. Comparison of the use of allogeneic blood transfusions.

\begin{tabular}{lccccc}
\hline USE OF BLOOD TRANSFUSIONS & COMB & IV1 & IV2 & TOP & $P$ \\
\hline Allogeneic blood transfusions & 15 & 20 & 9 & 23 & 0.044 \\
Total allogeneic + autologous blood transfusions & 24 & 35 & 23 & 30 & 0.198 \\
\hline
\end{tabular}

Allogeneic blood transfusions, substitution by red cell mass from a donor; autologous, substitution by the patient's own blood transfusion collected during pre-operative preparation. TOP, topical administration of TXA; COMB, combination of topical and intravenous administration of TXA; IV1, single intravenous administration; IV2, two intravenous doses of TXA; $P$, significance.

(450 mL) compared to topical administration $(635 \mathrm{~mL})$. A more detailed overview of perioperative loss is given in Table 2. Intravenous and combined administration lead to the significantly lowest total blood loss, including hidden loss, compared to isolated topical administration $(P<0.0001)$. Blood loss during surgery did not differ between cohorts $(P=0.268)$. A detailed overview of the results is given in Tables 2 and 3. A graphical representation of blood loss range is given in Fig. 2 and 3.

\section{Hidden blood loss}

Topical administration lead to the numerically highest hidden blood loss, i.e. loss to dead space, but a significant difference was found between TOP and IV2 $(P=0.010)$.
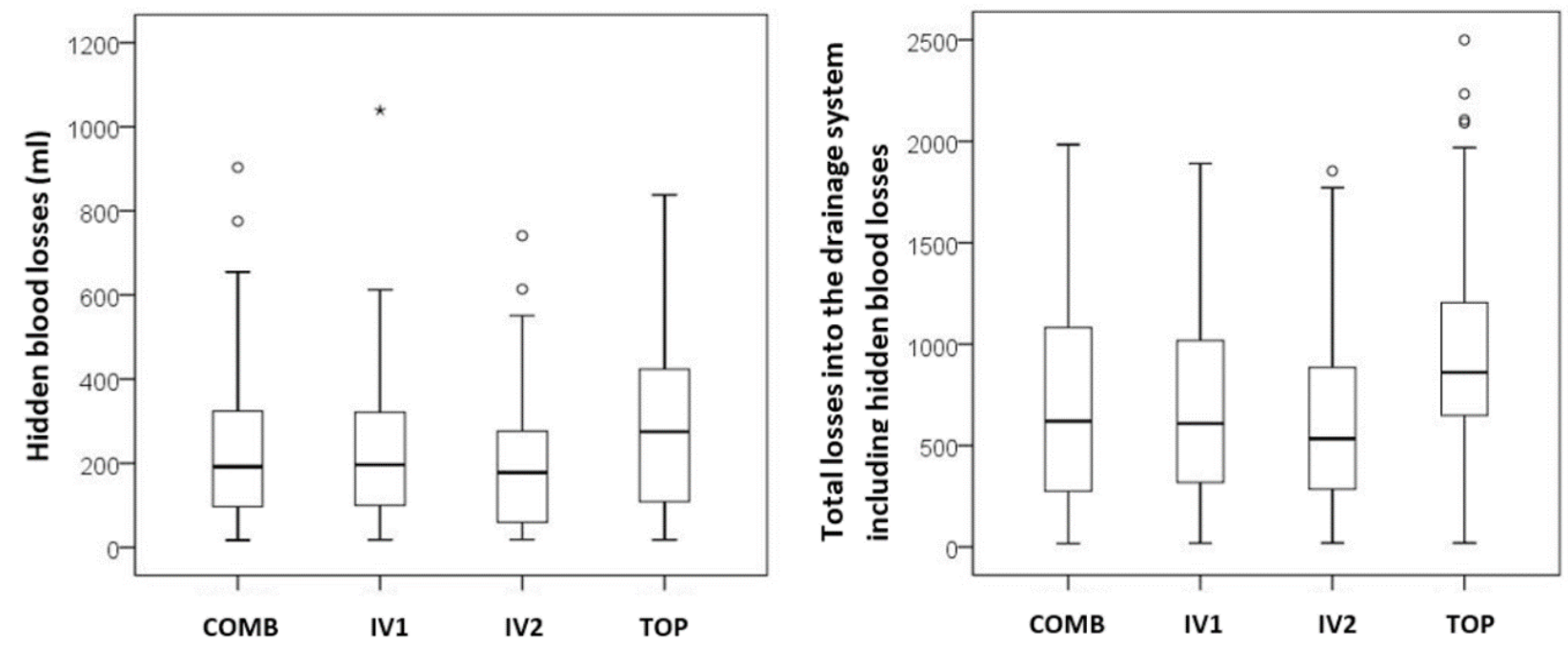

Fig. 4. Range of hidden blood loss during surgery (a) and in addition to postoperative blood loss to the drainage system (b) for each group.

TOP, topical administration of TXA; COMB, combination of topical and intravenous administration of TXA; IV1, single intravenous administration; IV2, two intravenous doses of TXA. 


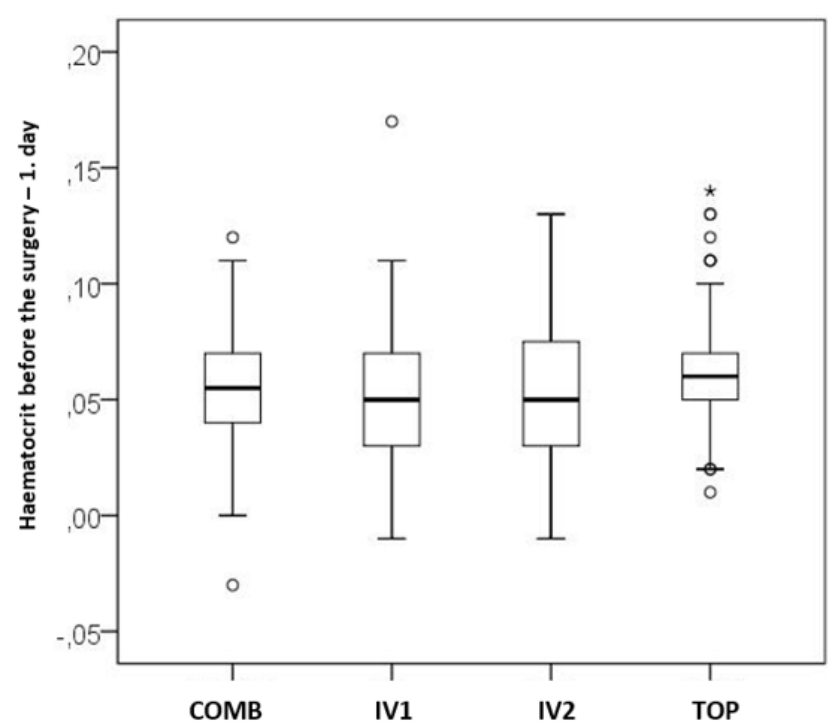

Fig. 5. Graphical representation of the difference between preoperative haematocrit and post-operative day 1.

TOP, topical administration of TXA; COMB, combination of topical and intravenous administration of TXA; IV1, single intravenous administration; IV2, two intravenous doses of TXA.

A comparison of the individual regimens in terms of the amount of hidden blood loss is shown in Fig. 4.

\section{Decrease in haemoglobin levels}

The mean postoperative haemoglobin level did not differ between groups. The distribution of differences in haemoglobin decrease from the preoperative value is shown in Table 4. Dunn's post hoc tests revealed differences in haemoglobin decrease only in the comparison of topical and combined TXA $(P=0.028)$ to topical and IV2 regimens $(P=0.033)$.

\section{Haematocrit}

A significantly greater difference was found between pre-operative haematocrit and haematocrit on the post- operative day 1 in the topical TXA regimen compared to a single intravenous administration $(P=0.010)$ and compared to the combined administration ( $P=0.048$, Fig. 5). Further, there was a significantly greater difference between haematocrit measured 3 and $6 \mathrm{~h}$ after the surgery when the topical administration regimen is compared to the IV1 regimen $(P=0.033)$. There were no differences in other haematocrit values.

\section{Consumption of allogeneic blood transfusions (EBR)}

The lowest use of blood transfusions was found after double intravenous administration of TXA (9/100). Conversely, the highest EBR use was found after topical administration (23/100). Details are given in Table 5.

\section{Post-operative complications (haematoma, wound secre- tion, early surgical revision)}

There were no differences in wound secretion and early postoperative revisions between groups. The lowest incidence of haematomas was found in IV2, but the difference was not statistically significant ( $P=0.291)$ compared to the other groups. There was a very low incidence of wound secretion cases (2-3\%) in all groups. There was also no difference in postoperative knee flexion measured at discharge $(P=0.332)$. Neither regimen showed a significantly higher frequency of early repeated surgeries $(P=0.905)$. A more detailed overview is given in Table 6 .

\section{Combined administration vs. other regimens}

We demonstrated that combined administration of TXA leads to significantly lower blood loss to the drainage $(P=0.004)$ and lower total blood loss including the drainage $(P=0.015)$ than topical administration of TXA. However, there was no significant difference compared to the intravenous regimens. We also found that combined administration of TXA leads to a significantly smaller haemoglobin decrease on day 1 compared to TOP $(P=0.028)$. No significant difference was found in comparison with the intravenous regimens.

Table 6. Comparison of hospitalization complications - haematoma, secretion, swelling, revision.

\begin{tabular}{|c|c|c|c|c|c|}
\hline & COMB & IV1 & IV2 & TOP & $P$ \\
\hline Haematoma occurrence & 24 & 22 & 16 & 27 & 0.291 \\
\hline \multicolumn{6}{|l|}{ Haematoma based on the size } \\
\hline up to $10 \times 10 \mathrm{~cm}$ & 6 & 7 & 4 & 12 & \multirow{3}{*}{0.265} \\
\hline over $10 \times 10 \mathrm{~cm}$ & 18 & 13 & 12 & 15 & \\
\hline almost the entire limb & 0 & 2 & 0 & 0 & \\
\hline Wound secretion & 3 & 2 & 2 & 3 & 1.000 \\
\hline Limb oedema & 23 & 22 & 23 & 24 & 0.990 \\
\hline Average duration of hospitalisation & 7.9 & 8.0 & 7.9 & 7.9 & 0.452 \\
\hline Knee flexion at the discharge (median) & $80(40-100)$ & $80(50-100)$ & $80(60-100)$ & $80(40-100)$ & 0.332 \\
\hline Surgical revision & 0 & 1 & 1 & 2 & 0.905 \\
\hline
\end{tabular}

TOP, topical administration of TXA; COMB, combination of topical and intravenous administration of TXA; IV1, single intravenous administration; IV2, two intravenous doses of TXA; $P$, significance. 


\section{Sex differences}

Total blood loss was $200 \mathrm{~mL}$ higher (on average) in men than women. The greatest difference was demonstrated with topical administration $(500 \mathrm{~mL}$ vs. 800 $\mathrm{mL})$. In women, there was a significant difference in total blood loss, including hidden loss, between different TXA regimens $(P=0.0001)$, while the difference was not significant in men. In men, there were significant differences in hidden blood loss $(P=0.019)$ and in the reduction of hidden loss in favour of dual intravenous administration $(P=0.020)$. In the context of the overall results, the lowest perioperative blood loss in both intravenous and combined administration was demonstrated in both sexes compared to topical administration alone (Table 7).

\section{DISCUSSION}

This prospective trial presents the comparison of the effects of four most common TXA regimens on blood loss reduction and use of blood transfusions in 400 patients following primary TKA. We found that the volume of blood loss increased in order: IV2, IV1 <COMB <TOP. Blood loss was higher in men by $200 \mathrm{~mL}$ on average than in women, with the greatest difference in the topical TXA regimen. The pattern seen in the whole cohort was maintained in both sexes, i.e., intravenous regimens lead to lower overall blood loss compared to combined and topical TXA. The differences in haemoglobin decrease depending on the route of TXA administration were not as clear as in blood loss. The lowest use of blood transfusions was seen with double administration of TXA.

According to recent meta-analyses of RCTs, intravenous administration is preferred for primary TKA due to its rapid onset of action and longer stability of therapeutic TXA concentration in the bleeding site ${ }^{2,11}$. Compared to topical administration, the effect of intravenous administration in the bleeding site is up to $5 \mathrm{~h}^{\text {longer }}{ }^{2}$. However, this is associated with higher systemic dose, which, in some patients, may induce VTE or other thrombotic complications. In our trial, we tested two forms of intravenous administration. In the first group, we administered a single intravenous dose of TXA (15 mg TXA $/ \mathrm{kg}$ ) at the induction of anaesthesia. In the second group, we administered an additional intravenous dose (15 mg TXA $/ \mathrm{kg}) 6$ hours after the first administration. Differences in blood loss volume between IV1 and IV2 were not significant in our trial, similarly to the differences in other endpoints. We may state, consistent with recent studies, that even the single dose regimen achieves an acceptable clinical benefit for patients undergoing primary TKA ( ref. $\left.^{14}\right)$. On the other hand, administration of 2x15 mg TXA $/ \mathrm{kg}$ did not increase the risk of serious perioperative complications according to our and other studies ${ }^{4}$. The effect was slightly better than the combined administration especially in the case of double administration, but this difference was not statistically significant.

Topical administration of TXA was the least effective approach for almost all endpoints (blood loss, haemoglobin decrease) in our trial. TXA is topically administered most often via irrigation of the surgical wound (intra-articular application) or less frequently via TXA infiltration directly into surrounding soft tissues, the so-called periarticular application ${ }^{2,15}$. However, recent meta-analysis has shown that the method of topical administration does not play a significant role ${ }^{2}$. The advantage of topical application is the maximal concentration at the application site, minimal resorption into the bloodstream. In our trial, we administered $2 \mathrm{~g}$ of TXA topically in a mixture with 50 $\mathrm{mL}$ of saline to irrigate the wound. Our previous trial also provided detailed reports on topical administration ${ }^{6}$. The reason for the lowest efficacy of topical administration in primary TKA is the fact that the topical effect is based on stopping the bleeding from soft tissues, not from spongy bone. Based on these findings, it seems most advantageous to combine both the routes in one procedure to stop bleeding from both soft tissues (topical administration) and bone marrow (intravenous administration). A recent meta-analysis ( 6 RCTs, 701 patients) showed very good results of combined administration on the reduction of total blood loss ${ }^{4}$. A combined administration was associated with less drainage to the drainage system and minimal haemoglobin decrease. On the other hand, the significant decrease in post-operative blood loss is not

Table 7. Comparison of perioperative blood loss by regimen and sex (data presented as median blood loss).

\begin{tabular}{|c|c|c|c|c|c|c|c|c|c|c|c|c|c|c|c|c|c|c|c|c|}
\hline & \multicolumn{5}{|c|}{$\begin{array}{l}\text { Total blood loss into drains } \\
\qquad(\mathrm{mL})\end{array}$} & \multicolumn{5}{|c|}{$\begin{array}{l}\text { Hidden blood loss } \\
\qquad(\mathrm{mL})\end{array}$} & \multicolumn{5}{|c|}{$\begin{array}{l}\text { Maximum blood loss } \\
(\mathrm{mL})\end{array}$} & \multicolumn{5}{|c|}{$\begin{array}{l}\text { Blood loss into drains in- } \\
\text { cluding hidden loss }(\mathrm{mL})\end{array}$} \\
\hline & $\sum_{0}^{\infty}$ & $\sum$ & $\sum$ & $\stackrel{0}{\varrho}$ & 2 & $\sum_{0}^{\oplus}$ & $\sum$ & $\sum$ & $\stackrel{0}{\ominus}$ & 2 & $\sum_{0}^{\oplus}$ & $\sum$ & $\sum$ & $\stackrel{0}{\ominus}$ & 2 & $\sum_{0}^{\infty}$ & $\sum$ & $\sum$ & $\stackrel{0}{\ominus}$ & 2 \\
\hline Female & $\frac{n}{n}$ & $\stackrel{\infty}{\sim}$ & ஓ & 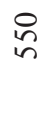 & 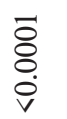 & $\bar{n}$ & $\cong$ & N & $\stackrel{0}{ \pm}$ & $\begin{array}{l}\infty \\
\cdots \\
0 \\
0\end{array}$ & § & $\stackrel{\circ}{\stackrel{2}{1}}$ & $\stackrel{\circ}{\circ}$ & $\stackrel{\circ}{\stackrel{n}{乙}}$ & ' & $\stackrel{n}{n}$ & $\frac{n}{*}$ & 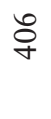 & $\stackrel{0}{2}$ & $\begin{array}{l}\dot{0} \\
\dot{0}\end{array}$ \\
\hline Male & $\stackrel{\circ}{6}$ & ஓூ & $\stackrel{n}{n}$ & ஓ & $\frac{\infty}{0}$ & $\stackrel{\infty}{\sim}$ & ภิ & $\stackrel{ナ}{\sim}$ & $\underset{\infty}{\infty}$ & $\stackrel{9}{0}$ & 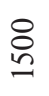 & ஓ & 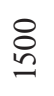 & $\stackrel{\circ}{\curvearrowright}$ & ' & aे & $\frac{b}{a}$ & $\stackrel{a}{n}$ & छ & $\begin{array}{l}\infty \\
0 \\
0\end{array}$ \\
\hline
\end{tabular}

TOP, topical administration of TXA; COMB, combination of topical and intravenous administration of TXA; IV1, single intravenous administration; IV2, two intravenous doses of TXA; $P$, significance. 
reflected in lower use of blood transfusions compared to other TXA routes. Importantly, TXA dose (>1.5 g) is critical for topical administration and the number of doses (i.e., more than one administration of TXA) plays a role in intravenous administration. Lin et al. published a systematic review and meta-analysis (15 studies, 1,495 patients) comparing the combined, intravenous and topical administration of TXA (ref. ${ }^{16}$ ). They report that combined TXA administration is mildly more effective in reducing blood loss compared to simple intravenous or topical administration of TXA. Blood loss was reduced in combined administration by almost $458 \mathrm{~mL}$. It was significantly more effective than intravenous administration in blood loss comparison $(P=0.034)$. According to Nielsen et al., the combined administration resulted in nearly $37 \%$ reduction in blood loss compared to simple intravenous administration within 24 hours after surgery and two days after the surgery ${ }^{17}$. Therefore, there is a question why the superiority of combined administration of TXA was not demonstrated in our and several other studies. One explanation may be the difficulty of standardizing the process of topical TXA administration. The effect of topical administration substantially depends on maintaining the TXA contact with the bleeding bed (tissue contact time) for at least five minutes ${ }^{18}$. It can also be assumed that the highest effect of topical TXA irrigation will be seen in surgeries when a tourniquet has been used since the beginning of the procedure. Bleeding starts once the tourniquet is released and the highest activation of fibrinolysis starts. At this point, the fastest haemostatic effect is achieved by the immediate topical antifibrinolytic effect of TXA administered directly on the bleeding bed. In our sample, the tourniquet was filled only during cementing of the implant (usually for 10-15 min). The formation of clot in topical administration may cause clogging of the drain and thereby mask the amount of visible blood loss to the drainage system. The difference should be reflected mainly in the decrease in haemoglobin or in the amount of hidden blood loss. Significant difference in hidden blood loss was shown only $12 \mathrm{~h}$ after the surgery in the IV2 regimen with an additional dose $6 \mathrm{~h}$ after the first administration.

Conversely, the difference in endpoints between topical and combined administration is clearly caused by a systemic dose of TXA, which is effective on its own. Jain et al. report a significant reduction of blood loss in combined use when compared to simple topical administration $(590.69 \pm 191.1$ vs. $385.68 \pm 182.5, P<0.001)\left(\right.$ ref. $\left.^{19}\right)$. The greater effect of combined TXA administration may be explained by the initial intravenous dose administered at the start of anaesthesia, thus covering the entire surgical period from the initial incision (especially for surgeries without a tourniquet). At the same time, the combined administration of TXA has a dual effect, as opposed to simple topical irrigation by affecting the systemic activation of fibrinolysis thanks to the intravenous dose ${ }^{18}$.

Sex could play an important role in the extent of blood loss. The number of women in TKA indications has long been higher than in men. The number of women in our cohort was also higher and their blood loss volume was on average $200 \mathrm{~mL}$ lower compared to men. However, the basic efficacy structure, i.e. from intravenous through combined to topical administration was seen in both sexes. A larger bleeding area can be assumed in men due to the size of the joint, affecting blood loss. Hormonal changes and/ or greater prevalence of obesity may play an important role in women. Rajesparan et al. showed a higher effect of intravenous dose of $1 \mathrm{~g}$ TXA in total hip arthroplasty in women $(P=0.05)$ than in men $^{20}$. There are no studies on total knee arthroplasty that address the different effect of TXA on sex basis.

We found no significant postoperative complications associated with TXA administration in this trial, similar to the previous trial on safety of TXA administration ${ }^{10}$. There were no thromboembolic complications in the study groups. Other meta-analyses have not shown any risk association with TXA administration. Thus, it can be stated that all tested routes of TXA administration are safe in selected group of patients.

\section{Study limitations}

The present study has certain limitations. We are aware of the fact that the data are crude estimates, particularly as concerns perioperative blood loss. In fact, there are no validated protocols enabling the volume of blood in drapes, dressings, wastes, etc., to be precisely measured. A second drawback is the difference between the physicians' approaches to the make-up for post-operative blood loss. Some physicians indicate administration of allogeneic blood at a haemoglobin level as high as 95-99 g/L whereas others are reluctant even when the level approaches 90 $\mathrm{g} / \mathrm{L}$ and less if the circumstances are otherwise favourable (young patient, no signs of the anaemic syndrome, etc.). Therefore, in theory the blood transfusion savings could be even higher.

\section{CONCLUSION}

The intravenous and combined regimens of TXA administration in primary TKA lead to a more significant reduction in blood loss than topical administration. The effect on blood loss into the drainage system can be expressed in the following order: IV2, IV1 <COMB <TOP. Intravenous and combined administration leads to the lowest total blood loss, including hidden loss, compared to topical administration $(P<0.0001)$. In contrast, the decrease in haemoglobin on the first postoperative day did not show the most advantageous intervention. The number of blood transfusions was highest in the group with topical TXA administration. In primary TKA, it is preferable to administer TXA intravenously in two doses or in a combined regimen. Simple topical administration is not as effective as other regimens and is indicated in case of contraindications to systemic administration. Follow-up studies should show whether it is possible to balance the effect of TXA in individual subgroups of patients according to the principles of precision medicine. 
Acknowledgement: This work was supported by the Ministry of Health of the Czech Republic - conceptual development of research organization (FNOL, 0098892 and RVO: 61989592 Palacky University IGA_LF_2020_016). Author contributions: JL: study design, data collection, analysis, and interpretation, writing; JG: funding, study design, data interpretation, writing, editing; LB: data collection, data interpretation; JZ: data analysis, data interpretation, writing.

Competing interests: The authors state there are no conflicts of interest regarding the publication of this article.

\section{REFERENCES}

1. Lee SY, Chong S, Balasubramanian D, Na YG, Kim TK. What is the Ideal Route of Administration of Tranexamic Acid in TKA? A Randomized Controlled Trial. Clini Orthop Relat Res 2017;475(8):1987-96.

2. Zhang S, Wang C, Shi L, Xue Q. Multi-route applications of tranexamic acid to reduce blood loss after total knee arthroplasty: a randomized controlled trial. Medicine 2019;98(30):e16570.

3. Xie J, Hu Q, Huang Q, Ma J, Lei Y, Pei F. Comparison of intravenous versus topical tranexamic acid in primary total hip and knee arthroplasty: An updated meta-analysis. Thromb Res 2017;153:28-36.

4. Xiong H, Liu Y, Zeng Y, Wu Y, Shen B. The efficacy and safety of combined administration of intravenous and topical tranexamic acid in primary total knee arthroplasty: a meta-analysis of randomized controlled trials. BMC musculoskeletal disorders 2018;19(1):321.

5. Kim YT, Kang MW, Lee JK, Lee YM, Kim Jl. Combined use of topical intraarticular tranexamic acid and rivaroxaban in total knee arthroplasty safely reduces blood loss, transfusion rates, and wound complications without increasing the risk of thrombosis. BMC musculoskeletal disorders 2018;19(1):227.

6. Lostak J, Gallo J, Vecera M, Spicka J, Langova K. [Local Application of Tranexamic Acid in Total Hip Arthroplasty Decreases Blood Loss and Consumption of Blood Transfusion]. Acta Chir Orthop Traumatol Cech 2017;84(4):254-62.

7. Han YH, Huang HT, Pan JK, Zeng LF, Liang GH, Liang HD, Yang WY, Guo D, Liu J. Is the combined application of both drain-clamping and tranexamic acid superior to the single use of either application in patients with total-knee arthroplasty?: A meta-analysis of randomized controlled trials. Medicine 2018;97(36):e11573.

8. Zhang H, He G, Zhang C, Xu B, Wang X, Zhang C. Is combined topical and intravenous tranexamic acid superior to intravenous tranexamic acid alone for controlling blood loss after total hip arthroplasty?: A meta-analysis. Medicine 2017;96(21):e6916.

9. Yuan X, Wang J, Wang Q, Zhang X. Synergistic effects of intravenous and intra-articular tranexamic acid on reducing hemoglobin loss in revision total knee arthroplasty: a prospective, randomized, controlled study. Transfusion 2018;58(4):982-8.

10. Lostak J, Gallo J, Slavik L, Zapletalova J, Balaz L. Monitoring of fibrinolytic system activity with plasminogen, $\mathrm{D}$-dimers and FDP in primary total knee arthroplasty (TKA) after topical, intravenous or combined administration of tranexamic acid. Biomed Pap Med Fac Univ Palacky Olomouc Czech Repub 2020,164(2):168-76 doi: 10.5507/bp.2019.034

11. Fillingham $Y A$, Ramkumar DB, Jevsevar DS, Yates AJ, Shores $P$, Mullen K, Bini SA, Clarke HD, Schemitsch E, Johnson RL, Memtsoudis SG, Sayeed SA, Sah AP, Della Valle CJ. The Efficacy of Tranexamic Acid in Total Knee Arthroplasty: A Network Meta-Analysis. J Arthroplasty 2018;33(10):3090-8.e1.

12. McPherson EJ, Woodson C, Holtom P, Roidis N, Shufelt C, Patzakis M. Periprosthetic total hip infection: outcomes using a staging system. Clin Orthop Relat Res 2002(403):8-15.

13. Shen HL, Li Z, Feng ML, Cao GL. Analysis on hidden blood loss of total knee arthroplasty in treating knee osteoarthritis. Chin Med J (Engl) 2011;124(11):1653-6.

14. Tzatzairis T, Drosos GI, Vogiatzaki T, Tilkeridis K, Ververidis A, Kazakos K. Multiple intravenous tranexamic acid doses in total knee arthroplasty without tourniquet: a randomized controlled study. Arch Orthop Trauma Surg 2019;139(6):859-68.

15. Pinsornsak $P$, Rojanavijitkul $S$, Chumchuen S. Peri-articular tranexamic acid injection in total knee arthroplasty: a randomized controlled trial. BMC Musculoskelet Disord 2016;17:313.

16. Burleson A, Guler N, Banos A, Syed D, Wanderling C, Hoppensteadt D, Rees H, Fareed J, Hopkinson W. Perioperative Factors and Their Effect on the Fibrinolytic System in Arthroplasty Patients. Clin Appl Thromb Hemost 2016;22(3):274-9.

17. Nielsen $C S$, Jans $O$, Orsnes $T$, Foss NB, Troelsen $A$, Husted $H$ Combined Intra-Articular and Intravenous Tranexamic Acid Reduces Blood Loss in Total Knee Arthroplasty: A Randomized, Double-Blind, Placebo-Controlled Trial. J Bone Joint Surg Am 2016;98(10):835-41.

18. Sun Q, Li J, Chen J, Zheng C, Liu C, Jia Y. Comparison of intravenous, topical or combined routes of tranexamic acid administration in patients undergoing total knee and hip arthroplasty: a meta-analysis of randomised controlled trials. BMJ open 2019;9(1):e024350.

19. Jain NP, Nisthane PP, Shah NA. Combined Administration of Systemic and Topical Tranexamic Acid for Total Knee Arthroplasty: Can It Be a Better Regimen and Yet Safe? A Randomized Controlled Trial. J Arthroplasty 2016;31(2):542-7.

20. Rajesparan K, Biant LC, Ahmad M, Field RE. The effect of an intravenous bolus of tranexamic acid on blood loss in total hip replacement. J Bone Joint Surg Br 2009;91(6):776-83. 